\title{
Plantas exóticas naturalizadas con potencial de invasoras en zonas periurbanas del Alta Amazonia Ecuatoriana
}

\author{
Naturalized exotic plants with invasive potential in peri-urban areas of the high Ecuadorian Amazon \\ Edison Carvajal ${ }^{1}$, Diego Gutiérrez ${ }^{1}$, Rubén Ledesma ${ }^{1}$ \\ ${ }^{1}$ Universidad Estatal Amazónica, Km 2 11/2 Vía Puyo-Tena, Puyo, Ecuador \\ *Correspondencia:ecaravjal@uea.edu.ec,dgutierrez@uea.edu.ec,rledezma@uea.edu.ec
}

Rec.: 01.05.2019. Acept.: 18.01.2020.

Publicado el 30 de junio de 2020

\begin{abstract}
Resumen
$\mathrm{L}^{\mathrm{as}}$ as especies exóticas invasoras son la segunda causa de pérdida de biodiversidad en el planeta. Adicionalmente la globalización y el cambio climático están favoreciendo su entrada en áreas poco humanizadas en el pasado como es la amazonia ecuatoriana. La cabecera cantonal del Puyo tiene numerosos vegetales exóticos ya reportados en otros lugares del mundo como invasores, que aquí han sido observados e identificados para aportar listados e información acerca de cuáles especies naturalizadas pueden en el futuro pasar a ser invasoras bajo los escenarios de cambio climático, y así mismo proponer de entre ellas a cuatro especies exóticas con el mayor potencial de comportarse como invasoras con el clima actual. El trabajo de campo fue realizado en el entorno periurbano de Puyo, identificando las especies con muestras en herbario, también a través de consultas bibliográficas y online, reportando numerosas especies exóticas naturalizadas que se organizaron jerárquicamente y se seleccionaron por sus atributos para ser invasoras potenciales o actuales, identificando 15 especies exóticas naturalizadas con potencial de ser invasoras bajo los escenarios de cambio climático destacando 4 especies vegetales que ya son o pueden convertirse en el futuro en un serio problema para la biodiversidad y la economía amazónica ecuatoriana. Con este trabajo se espera que las autoridades ambientales y los habitantes tomen conciencia de su presencia y se apliquen medidas para su erradicación temprana.
\end{abstract}

Palabras claves: biodiversidad, especies, introducidas, naturalizadas, cambio climático.

\begin{abstract}
Tnvasive alien species are the second cause of biodiversity loss on the planet. Additionally, globalization and climate change are favoring its entry into areas that have not been humanized in the past, such as the Ecuadorian Amazon. The cantonal capital of Puyo has numerous exotic plants already reported in other parts of the world as invaders, which here have been observed and identified to provide listings and information about which naturalized species in the future can become invasive under the scenarios of climate change. , and also propose among them four exotic species with the greatest potential to behave as invasive with the current climate. The fieldwork was carried out in the periurban environment of Puyo, identifying the species with herbarium samples, also through bibliographic and online consultations, reporting numerous naturalized exotic species that were organized hierarchically and selected for their attributes to be potential invaders or current, identifying 15 naturalized exotic species with the potential to be invasive under the climate change scenarios highlighting 4 plant species that are already or can become in the future a serious problem for biodiversity and the Ecuadorian Amazonian economy. With this work it is expected that the environmental authorities and the inhabitants become aware of their presence and apply measures for their early eradication.
\end{abstract}

Keywords: biodiversity, species, introduced, naturalized, climate change. 


\section{Introducción}

$\mathrm{L}$ as especies exóticas invasoras (EEI) son la segunda causa de pérdida de especies biológicas en el mundo (Aguilar, 2005) y sus efectos perjudiciales sobre la biodiversidad ya fueron señalados en el Convenio sobre la Diversidad Biológica (CDB, 1992), el cual entró en vigor el 29 de diciembre de 1993. Sin embargo, al mismo tiempo dichas especies ocasionan cuantiosas pérdidas en agricultura y otras áreas como la salud humana o sobre el valor paisajístico del lugar, que a la larga perjudican al ecoturismo y la economía de una región (Hernández, 2008). Su acción negativa sobre la biodiversidad vegetal se manifiesta principalmente debido a que compiten por espacio, agua y nutrientes y de esta manera por un lado desplazan a las nativas (Aguilar, 2005), porque en ese nuevo territorio las EEI no tienen los mecanismos de control que sí había en su lugar de origen y por el otro a la larga, las EEI al desplazar las especies nativas, perjudican la sucesión ecológica que de forma natural acontece en ambientes perturbados y con ello eliminan la regeneración natural de los ecosistemas terrestres, causando daños permanentes. Otros efectos documentados sobre las plantas nativas son daños en su reproducción por competencia con insectos polinizadores (caso de Lantana cámara L., Verbenaceae y su similitud floral con orquídeas del género Epidendrum como E. radicans Pav. ex Lindl.) y porque como ha sucedido con el árbol de papel foráneo Polylepis racemosa Ruiz \& Pav., Rosaceae llevada a hábitats donde existen especies nativas del mismo género, pueden originar fenómenos de hibridación con las propias del lugar, afectando la biodiversidad a largo plazo (MAE, 2011; Segovia et al., 2010). Muchas EEI dependiendo del nuevo lugar en el que se hallen, pueden llegar a convertirse en transformadoras del hábitat y así por sí solas pueden modificar las condiciones biofísicas y las comunidades de los hábitat terrestres y acuáticos (Hernández, 2008).

La humanidad depende de las plantas en muchos sentidos y cualquier colonización de un nuevo territorio conlleva la introducción de especies vegetales; sean alimenticias, medicinales, maderables y por último aquellas de valor ornamental (Ojasti, 2001) y a veces pueden asilvestrarse como es el caso de la guayaba o algunas especies de Guaba (Inga sp. Leguminosae), traídas al Ecuador probablemente junto con las primeras comunidades humanas que hace 15000 años atravesaron el istmo de Panamá y son consideradas plantas nativas o inmigrantes de tiempos pretéritos (Ojasti, 2001). Según Herrera y colaboradores (2016) para que una especie foránea sea EEI, previamente deben superarse ciertas barreras, pero una vez llega al nuevo lugar, la especie se considera introducida. Si el nuevo clima es adecuado para la planta, ésta se desarrolla bien y puede dar semillas o propágulos que se dispersen y colonicen las zonas cercanas pasando a ser una especie establecida (Williamson y Fitter, 1996). Con el tiempo algunas de estas especies se mezclan con las plantas nativas y quedan instaladas en una nueva región, considerándose entonces como especie exótica naturalizada (EEN). Finalmente, y según la Convención sobre Diversidad Biológica y la UICN, una especie se define como invasora o EEI cuando se propaga desplazando a las nativas y por ende amenazando los ecosistemas, hábitats y/o especies y a la vez causando daños económicos y ambientales (Lowe et al., 2000).

La Alta Amazonía Ecuatoriana (AAE) en la provincia de Pastaza, continuaba sin intervención humana importante incluso después de los años 60 de siglo XX, debido a que el abrupto descenso de las montañas andinas desde la ciudad de Baños, carecía de vías adecuadas y por ello su acceso era complicado; pero desde las mejoras viales de los años 90 su colonización y a su vez las modificaciones del medio van incrementando y con ello también la llegada de nuevas especies foráneas que acaben naturalizándose (Trombulak y Frissell, 2000). La globalización y el desarrollo económico de Ecuador han acelerado este proceso y se espera que poco a poco estas especies exóticas naturalizadas (EEN) se conviertan en frecuentes y probablemente con el paso del tiempo algunas a su vez se conviertan en especies problemáticas para la AAE y que ocasionen daños ambientales y/o económicos, por lo que su catalogación y divulgación a las autoridades ambientales y al público en general, adquiere una importancia para esta región ecuatoriana que contiene una alta diversidad biológica.

\section{Materiales y métodos}

\section{Sitio de estudio}

El sitio elegido para la catalogación de especies exóticas naturalizadas, fueron los lugares antropizados del casco urbano y zonas periurbanas de la cabecera cantonal de Puyo, cantón Pastaza $\left(1^{\circ} 29^{\prime} 25^{\prime \prime} \mathrm{S} 78^{\circ} 00^{\prime} 08^{\prime \prime} \mathrm{O}\right.$, 950 m.s.n.m), ubicada en la AAE y que recibe más de 4000 m.m de lluvia anual sin estación seca. Esta ciudad tiene una expansión reciente y muy rápida por lo que su casco urbano está modificándose constantemente. El área de estudio estaría limitando al Noroeste por el paso perimetral de la vía Ambato-Tena en la Troncal Amazónica E-45, al Este y Noreste por el Rio Puyo y al Sur por el límite de la Parroquia Tarqui (Figura 1).

\section{Identificación de plantas y atributos usados para su selección}

Por medio de observaciones de campo durante los años 2015 al 2017, fotografiando las muestras para identificación botánica en herbario y también a través de consultas bibliográficas y online (tropicos.org, theplantlist. org), fueron determinadas numerosas especies exóticas 


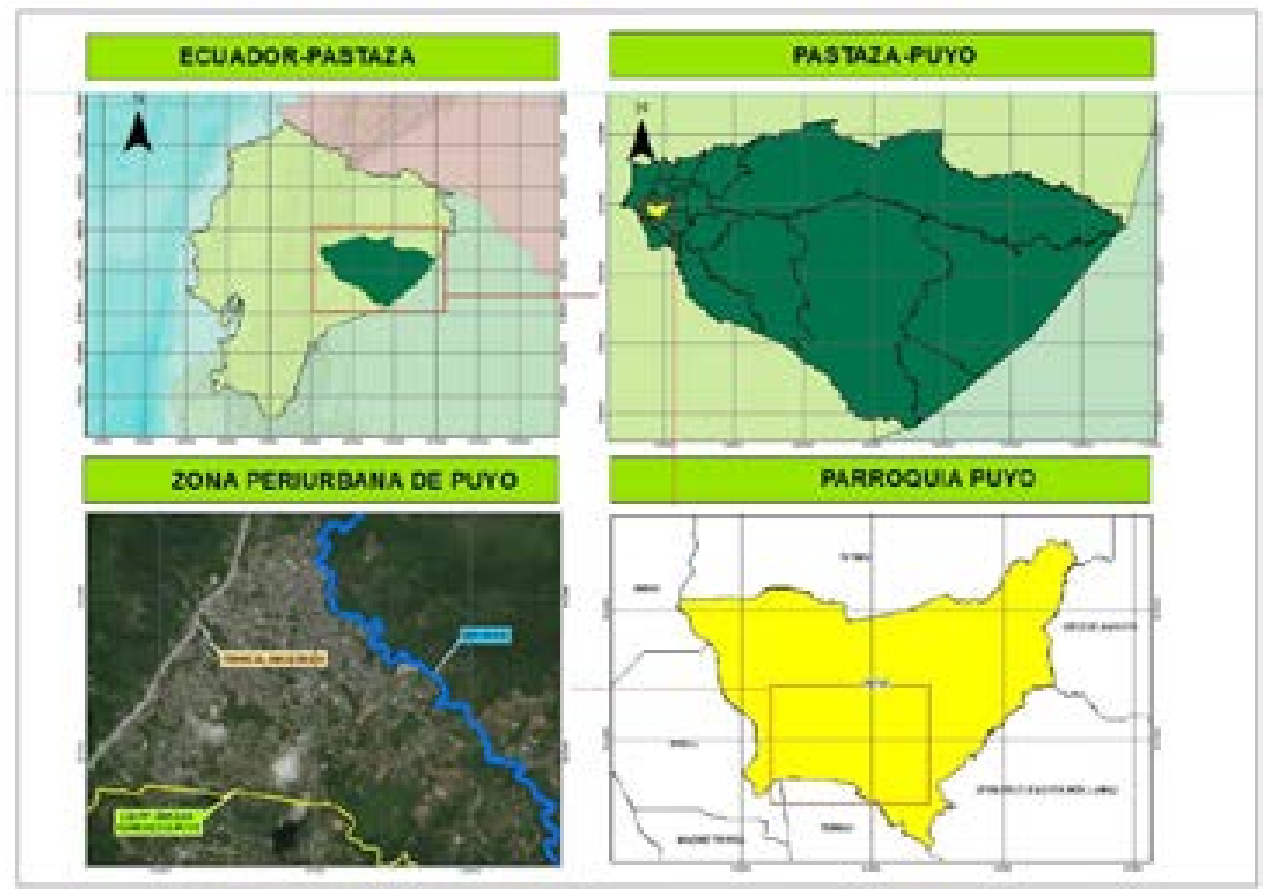

Figura 1. Ubicación geográfica del área periurbana de Puyo

naturalizadas (EEN) en el área urbana de Puyo. Aquellas especies vegetales exóticas útiles ya trasladadas por las primeras comunidades humanas que llegan al Ecuador, en nuestro estudio se consideraron como nativas o inmigrantes de tiempos pretéritos de acuerdo con Ojasti (2001) y por tanto no formaron parte de los inventarios de este estudio.

Después de su identificación botánica y de constatar su presencia en más de un sitio, así como su capacidad de reproducirse en la zona de estudio; todas las especies halladas se organizaron jerárquicamente en función de su potencial invasor, empleando como atributos de selección en primer lugar la bibliografía, adquiriendo más relevancia cuando se encontraban en la lista de las 100 peores especies invasoras de acuerdo a la publicación realizada por el Grupo Especialista de Especies Invasoras GEEIE (Lowe, et al., 2000) o en aquellos trabajos que indican cómo esa especie en cuestión ya está causando problemas en otras áreas de Ecuador (MAE, 2011; Herrera, 2016) y de América Latina. El segundo atributo en importancia fue su forma de vida y altura total de la planta, de acuerdo con Williamson y Fitter (1996), porque en la AAE los terófitos (plantas herbáceas) con menos de $2 \mathrm{~m}$ de altura máxima en general no son buenos competidores, ya que en esta región con lluvias constantes todo el año la mayoría de vegetales pioneros ya sean nativos o inmigrantes de tiempos pretéritos, en poco tiempo pueden superar la altura de las nuevas plantas exóticas impidiendo su expansión y evitando que pasen a ser EEI. En último lugar se tuvo en cuenta la resistencia a sequias (extrapolada a partir de su origen y del área que ocupa a nivel mundial) lo cual le permitiría soportar futuros escenarios de cambio climático proyectados para la AAE y si su capacidad de dispersión es por propágulos (vegetativa), sexual o ambas, así como producción y dispersión de semillas viables constatada por la observación de frutos y/o plántulas en sitios urbanos. Con estos datos fueron seleccionadas las 15 especies mostradas en la cuadro 1 y para construir la cuadro 2 , fueron seleccionadas las cuatro EEN que representan un peligro real y un alto potencial de expansión y establecimiento en esta zona.

\section{Resultados y discusión}

Tos ambientes ruderales de esta ciudad poseen cifras ${ }_{\text {superiores a }} 50$ especies exóticas naturalizadas con herbáceas de las familias como Poaceae, Euphorbiaceae y Asteraceae muy bien representadas; pero más de la mitad de ellas no son muy competitivas porque solo alcanzan $0,5-$ $1 \mathrm{~m}$ de altura y con ese tamaño de la parte aérea, carecen de potencial para desplazar a la vegetación natural, que en poco tiempo debido a las abundantes lluvias y temperaturas de la zona, pueden alcanzar una altura considerable y evitar el ser desplazadas por las exóticas de nueva introducción.

Entre aquellas especies exóticas del área periurbana de Puyo que la bibliografía contempla como potencial EEI, pueden encontrarse a la lenteja de agua (Lemna gibba L.) y varios taxones del género Oxalis que por su fácil formación de propágulos hoy día han colonizado casi todo el trópico. La primera especie, denominada comúnmente lenteja de 
agua es acuática, por ello fácilmente dispersada a grandes distancias por aves migratorias y que se han observado en algunos esteros, mientras que varias especies de Oxalis llegan en macetas como acompañante de ornamentales adquiridas en otras ciudades de la zona andina donde están bien establecidas y se instalan en zonas ajardinadas urbanas para después ser dispersadas vegetativamente con la remoción de tierras, pero dichas especies, aunque fueron observadas poblaciones instaladas en los años precedentes, actualmente no muestran un establecimiento definitivo en la zona, lo que hace pensar que existe algún impedimento en su ciclo biológico, seguramente debido a las particulares condiciones ambientales con altas precipitaciones y muy pocas horas de luz solar que existen en la Alta Amazonía Ecuatoriana. Otro caso observado es la llegada en macetas destinadas a zonas ajardinadas del diente de león (Taraxacum officinale L.) que, aunque podría instalarse gracias a su elevado potencial de establecimiento por su gran producción de semillas y la eficiencia en su dispersión que le ha permitido colonizar casi todo el globo terráqueo (Mendoza y Koleff, 2014), en el área de Puyo esta especie no perdura y su presencia desaparece al cabo de pocos años. Como se observa en la tabla 1 dentro de las herbáceas solo se ha considerado a la hierba "coniza" al ser bastante frecuente en toda la AAE y capaz de formar numerosas plantas invadiendo en una alta proporción los terrenos desnudos del área estudiada (Cuadro1).

Como se indicó, existen numerosas especies exóticas naturalizadas en los ambientes perturbados de Puyo, pero en el presente trabajo las especies consideradas como EEN y EEI exclusivamente se refieren a introducciones históricas recientes o bien plantas causantes de invasiones en otras zonas del país o del trópico confirmadas con la bibliografía y que alcancen un tamaño cercano a $2 \mathrm{~m}$, por ello las herbáceas de pequeño porte como por ejemplo Sphagneticola trilobata L. (Asteraceae), observada en algún punto del área de estudio y aunque forma parte de la lista de las 100 especies exóticas invasoras más dañinas del mundo (Lowe et al., 2000 ), por su escasa altura, no se han incluido en las tablas. Como ya se ha indicado, tampoco se han considerado a las plantas útiles exóticas naturalizadas.

La única especie alimenticia que sí fue pertinente para este trabajo es la mora silvestre Rubus niveus, Rosaceae (Cuadros 1,2) donde se aprecian los 9 foliolos que la distinguen de otras especies semejantes y probablemente también naturalizadas en Pastaza como Rubus glaucus Benth., debido a que gracias a su alta producción de semillas y a la fácil dispersión por aves, ya comienza a ser frecuente en zonas urbanas y huertos abandonados (Soria. 2002) y se considera por las autoridades ambientales una de las principales especies invasoras en el país (MAE, 2011); siendo en Galápagos junto con Lantana cámara (que solo se incluyó en el Cuadro 1 porque muestra una menor presencia), las dos principales EEI de las islas y sometidas a programas de erradicación (Buddenhagen, 2006). L. camara empieza a ser usada como cerca viva y ornamental en Puyo y también comienza a naturalizarse gracias a su dispersión por aves (cuadro 1). Aunque esta última especie o Eichhornia crassipes, Pontederiaceae (Jacinto de agua) sean especies sudamericanas, en la AAE claramente se encuentran fuera de su nicho original, por ello y en base a la bibliografía (Hernández, 2008; Herrera, 2016), sí se consideraron pertinentes para este trabajo y por ello también fueron reflejadas en las tablas. El Tulipán africano (Spathodea campanulata), La acacia forrajera (Leucaena leucocephala), la Caña de Castilla (Arundo donax), la supirrosa (L. camara) y el Jacinto de agua (E. crassipes) son las cinco especies que están recogidas por la IUCN dentro de las 100 peores especies invasoras del mundo (Lowe et al., 2000) y a la vez también están incluidas en la tabla 11 . Al revisar dicha tabla podemos comprobar que el hábito más frecuente fue de hemicriptofitas, es decir, plantas perennes con rizomas, que si bien es cierto que en otras latitudes alguna de ellas no suele alcanzar más de 1.5 $\mathrm{m}$, en el clima tropical de la zona de estudio con lluvias frecuentes durante todo el año, algunas pueden superar sin problema los $2 \mathrm{~m}$ de altura siendo actualmente ya una EEI muy agresiva, como es el caso de la flor de muertos Hedychium coronarium (Cuadros 1,2) con una aparición en casi todos los pastizales y bordes de carretera de la zona periurbana. Las 2 especies arbóreas halladas en el área y que forman parte del Cuadro 1 fueron $S$. campanulata, (tulipán africano) y L. leucocephala (acacia forrajera) y ninguna de ellas muestra una buena producción de semillas viables, por lo que no tienen alta frecuencia de aparición en la zona (obs. Pers.)

Sin embargo, ambos vegetales alcanzan una altura considerable y al mismo tiempo se encuentran catalogadas dentro de las 100 peores especies invasoras del mundo (Lowe et al., 2000), sumado a todo esto y en base a su lugar de origen y distribución, se puede suponer que si el cambio climático en la AAE conduce a un clima que se tornase más seco, su reproducción, expansión y aumento de dispersión será posible. Otras especies como E. crassipes y Molineria capitulata se incluyeron en los cuadros 1 y 2 de este trabajo, ya que, aunque tienen baja frecuencia y porte, $M$. capitulata con sus profundos rizomas invade todo el espacio disponible y tolera ambientes sombreados, tanto ella como $E$. crassipes presentan un claro potencial invasor capaz de perdurar allí donde llegan y por estas razones forman parte de las 4 especies recopiladas en el Cuadro 2 que incluso hoy día deben considerarse problemáticas para la AAE. También entre los vegetales invasores actualmente escasos, y que forma parte del listado del Cuadro 2, se encuentra la mora silvestre, que tiene la capacidad de invadir rápidamente campos de cultivos los cuales quedan inutilizados y cuya recuperación es muy costosa (Herrera 
Plantas exóticas naturalizadas con potencial de invasoras en zonas periurbanas del Alta Amazonia

Ecuatoriana

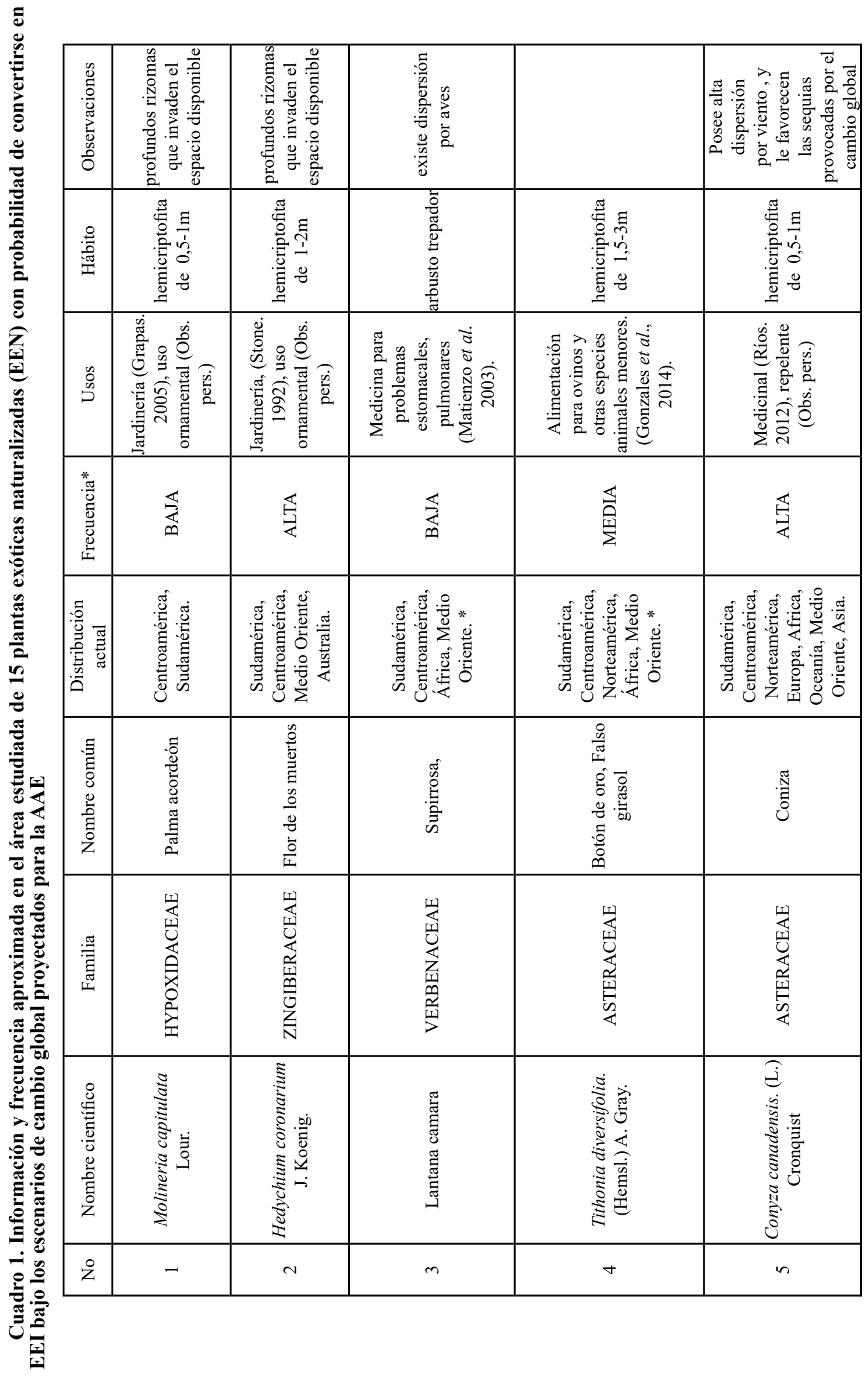




\begin{tabular}{|c|c|c|c|c|}
\hline 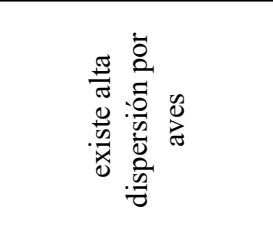 & 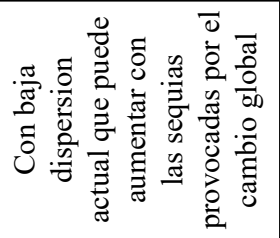 & 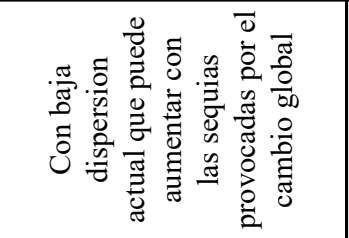 & 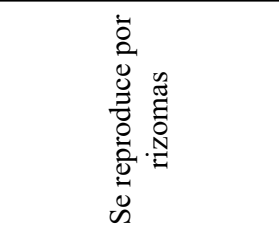 & 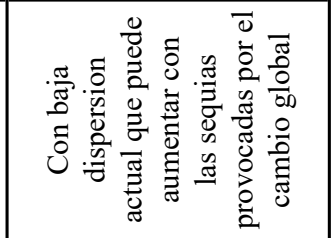 \\
\hline 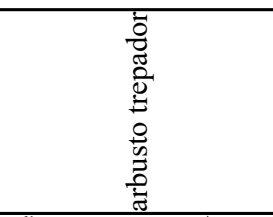 & 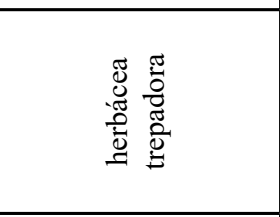 & 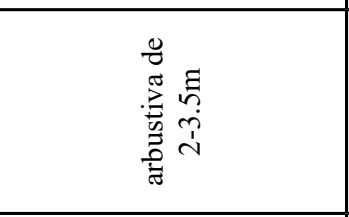 & 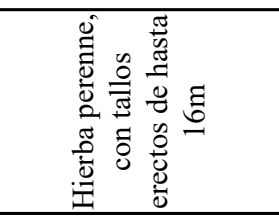 & 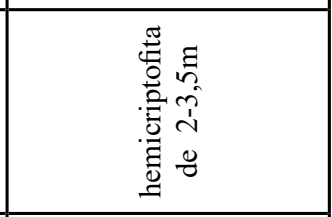 \\
\hline 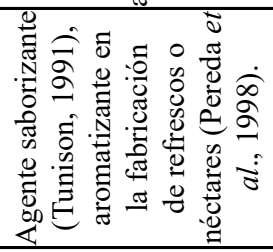 & 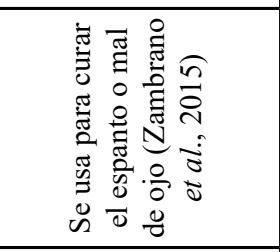 & 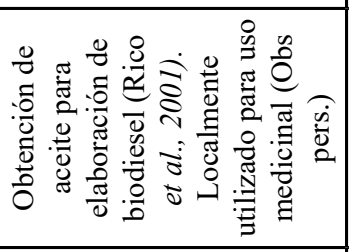 & 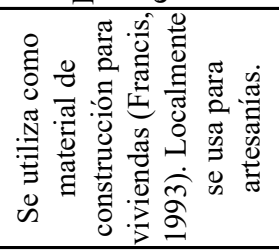 & 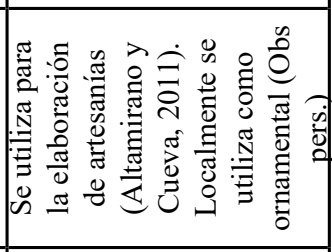 \\
\hline $\begin{array}{l}\overleftrightarrow{\Xi} \\
\stackrel{1}{\Sigma}\end{array}$ & 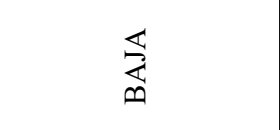 & 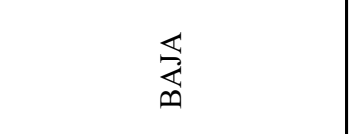 & $\begin{array}{l}\overleftrightarrow{\Xi} \\
\stackrel{\Xi}{\Sigma}\end{array}$ & 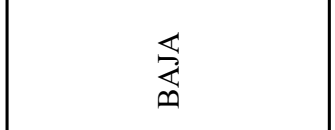 \\
\hline 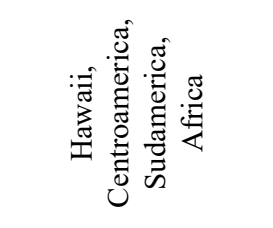 & 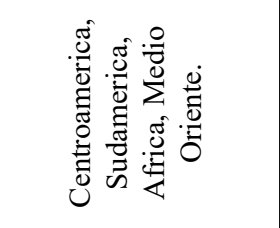 & 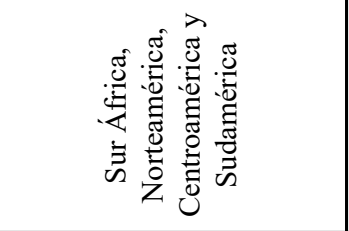 & 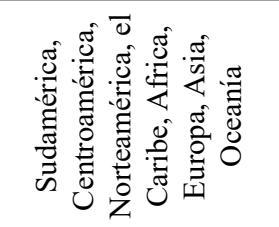 & 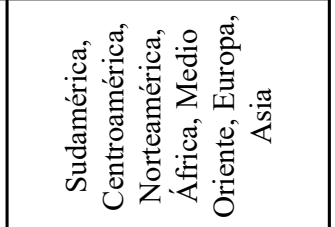 \\
\hline \multirow[t]{2}{*}{ 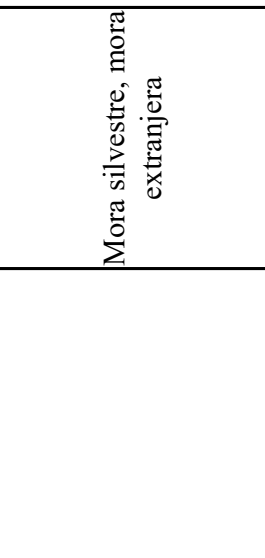 } & $\begin{array}{l}0 \\
0 \\
0 \\
0 \\
0 \\
0 \\
0 \\
0\end{array}$ & 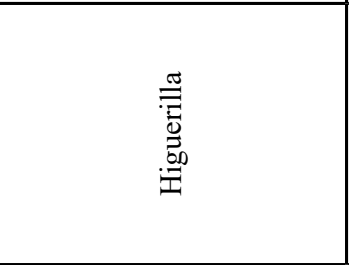 & 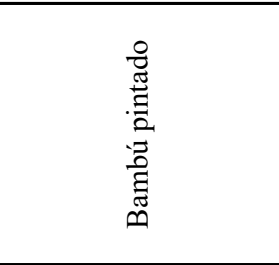 & 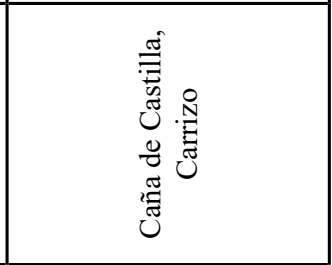 \\
\hline & 窀 & 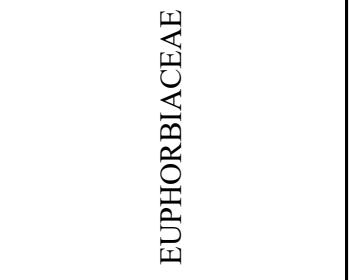 & 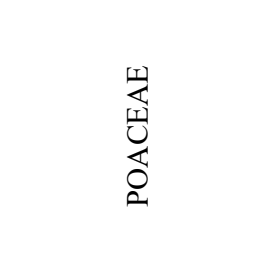 & 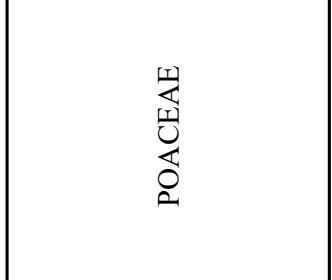 \\
\hline 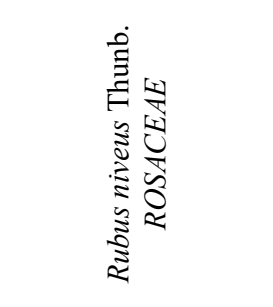 & 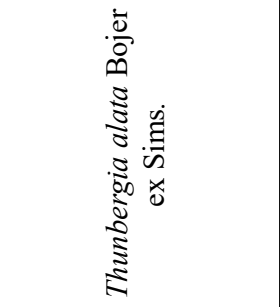 & 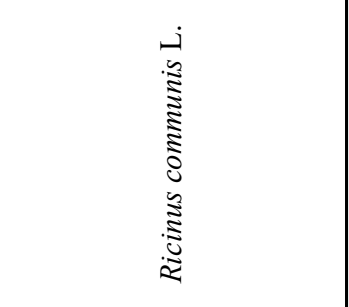 & 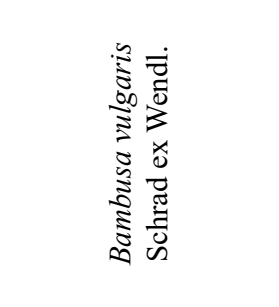 & 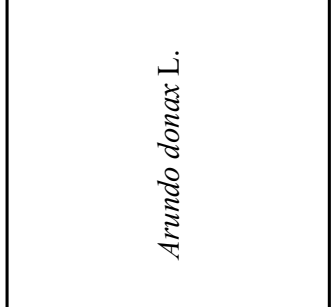 \\
\hline 0 & $r$ & $\infty$ & $a$ & 우 \\
\hline
\end{tabular}




\begin{tabular}{|c|c|c|c|c|c|c|}
\hline \multicolumn{2}{|c|}{ 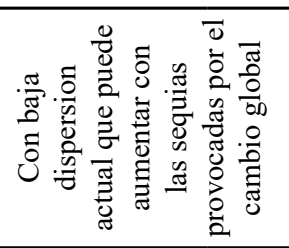 } & 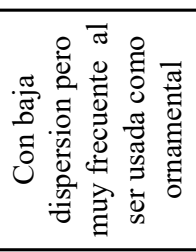 & & & & \multirow{3}{*}{ 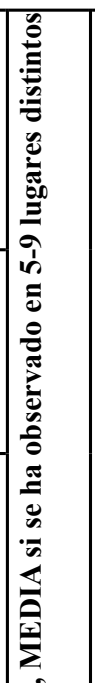 } \\
\hline & & 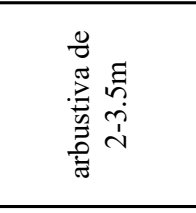 & 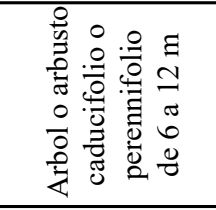 & 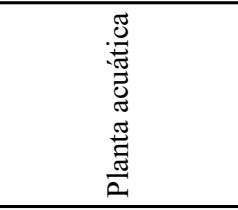 & 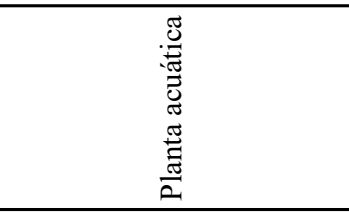 & \\
\hline \multicolumn{2}{|c|}{ 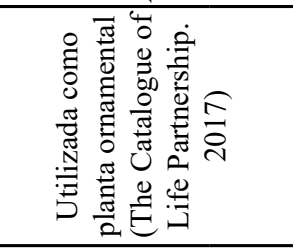 } & 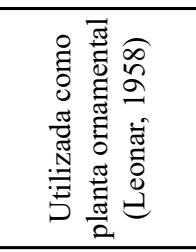 & 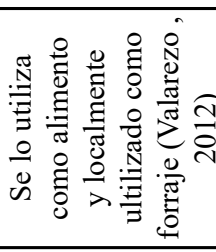 & 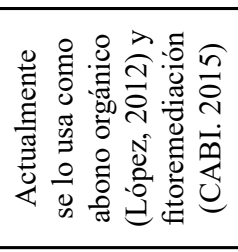 & 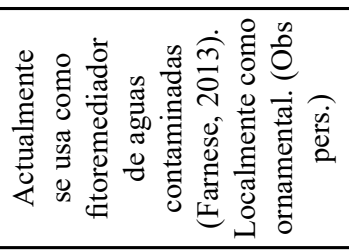 & \\
\hline \multicolumn{2}{|c|}{ 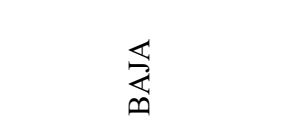 } & $\underset{⿱ 乛}{\rightleftarrows}$ & $\underset{\infty}{\overleftrightarrow{\Phi}}$ & 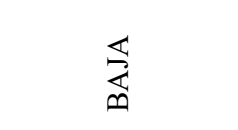 & 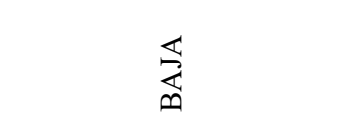 & $\begin{array}{l}\bar{\sigma} \\
\bar{d} \\
\bar{\delta} \\
\tilde{y}\end{array}$ \\
\hline \multicolumn{2}{|c|}{ 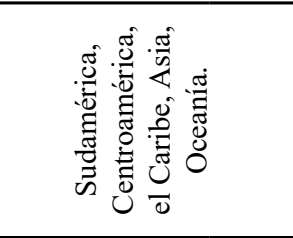 } & 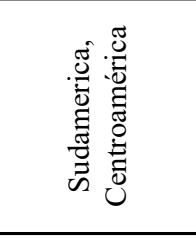 & 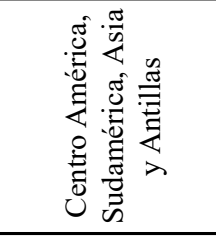 & 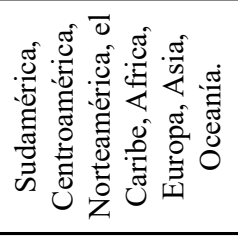 & 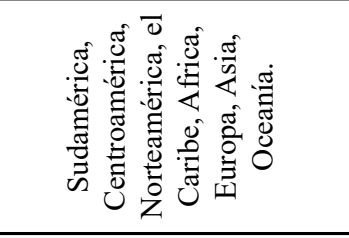 & 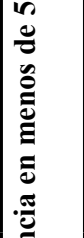 \\
\hline \multicolumn{2}{|c|}{ 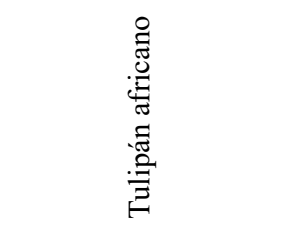 } & 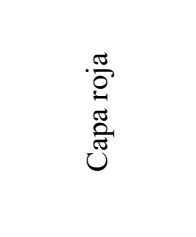 & 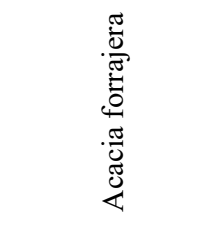 & 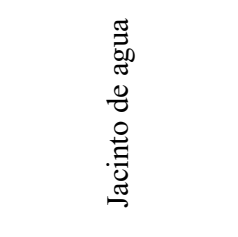 & 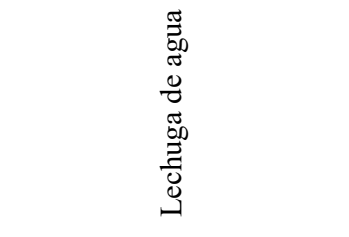 & \multirow{4}{*}{ 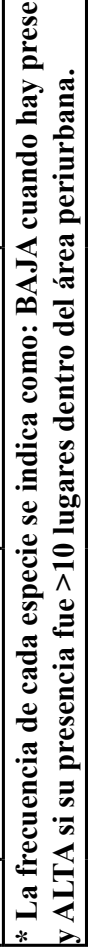 } \\
\hline & & 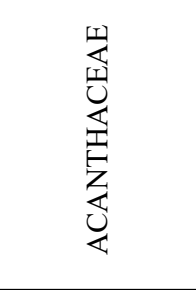 & 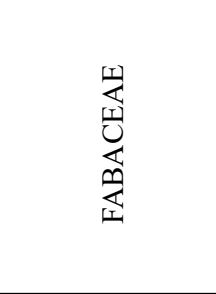 & 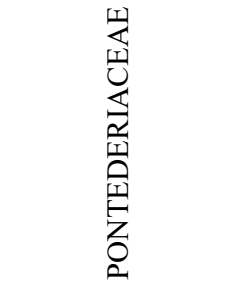 & 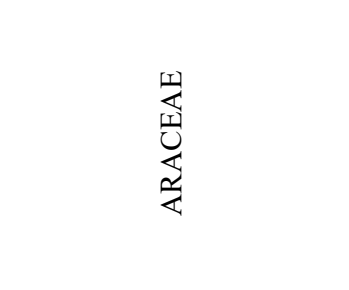 & \\
\hline 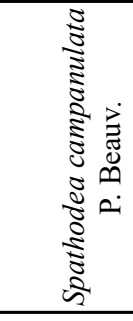 & 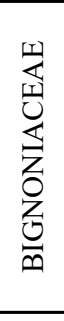 & 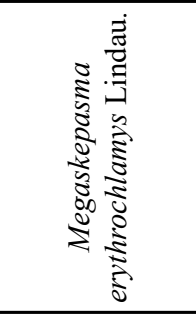 & 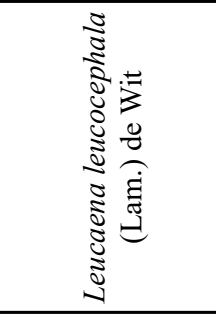 & 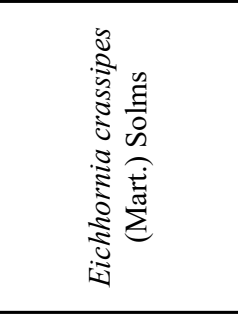 & 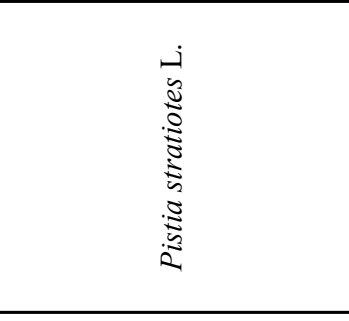 & \\
\hline \multicolumn{2}{|c|}{$=$} & $\simeq$ & $\stackrel{2}{=}$ & $\Xi$ & $\cong$ & \\
\hline
\end{tabular}


Carvajal et al., 2020

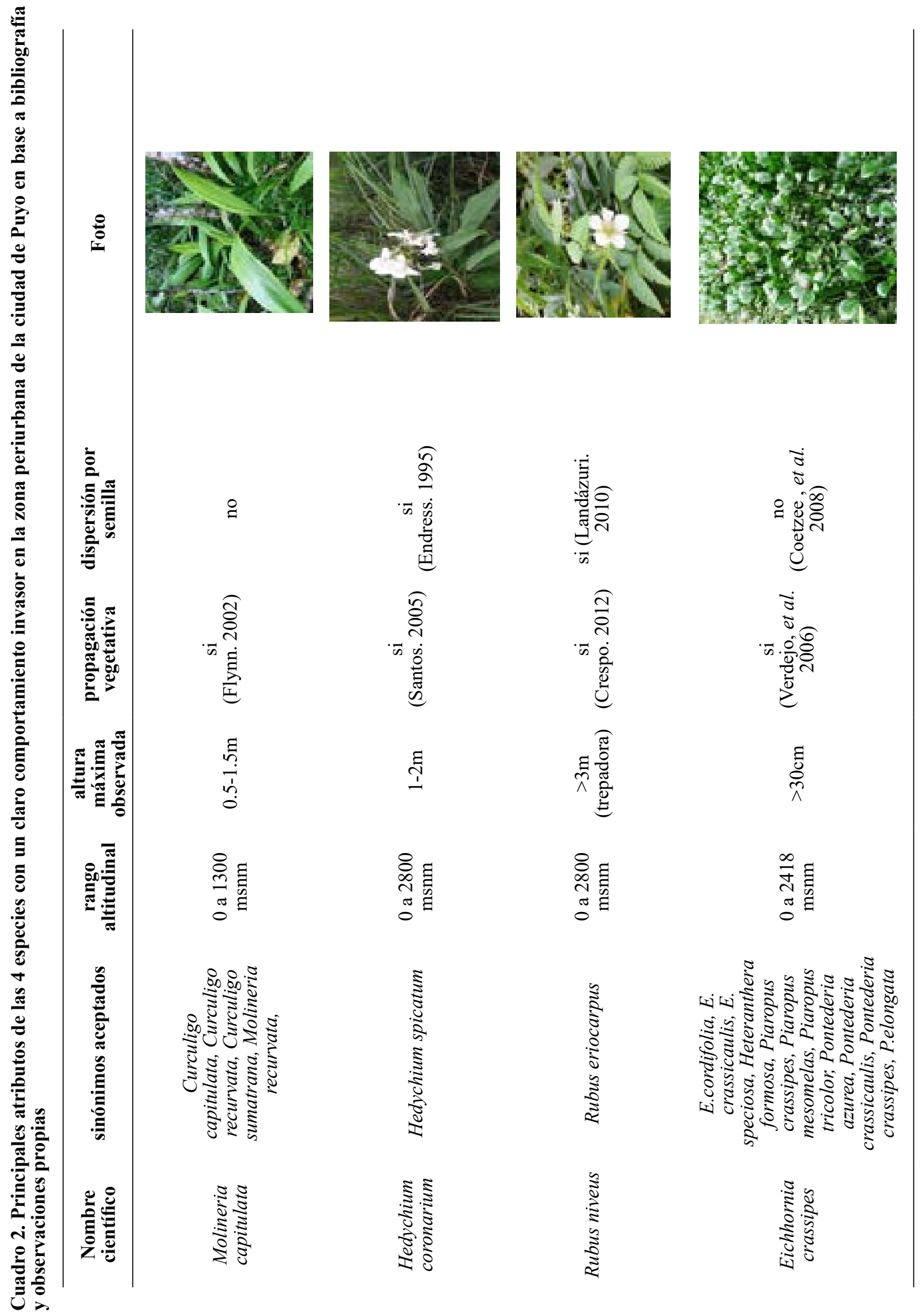


a)
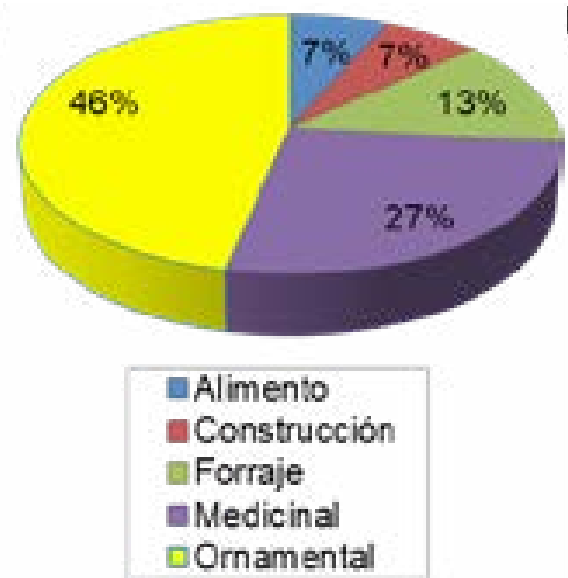

b)

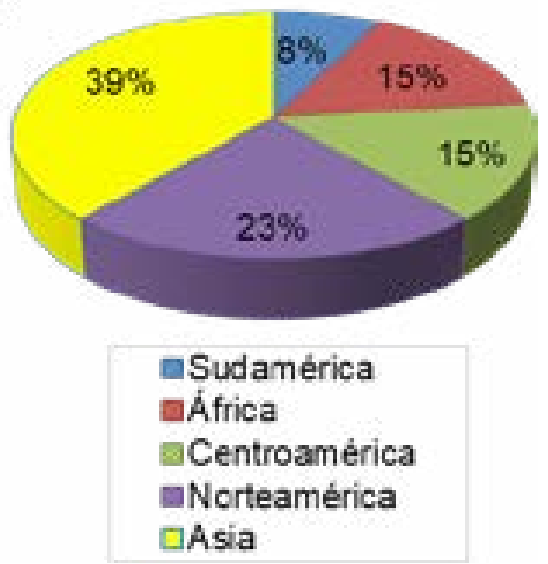

Figura 2. Análisis de las 15 plantas exóticas naturalizadas (EEN) del Cuadro 1, con los principales usos para el hombre (a) y lugar de origen según la bibliografía (b) catalogadas para la ciudad y área periurbana de Puyo (Pastaza)

Los resultados indican que la mayoría de los usos dados por el hombre a estas especies correspondieron con su empleo como planta ornamental (27\%) seguido del uso medicinal con un 27\% (Cuadro1, figura 2a) esto sugiere cuales fueron los motivos de introducción en la zona, si bien es cierto que no se puede descartar la reintroducción fortuita desde otras zonas del país en el caso de la flor de los muertos, que está muy extendida en toda la región amazónica de Ecuador y otros países sudamericanos (Herrera et al., 2016). El lugar originario de las EEN observadas en esta zona fue principalmente Asia con 5 especies (39\%) seguido de Norteamérica que con 3 especies obtuvo un 23\% (Figura $2 b$ ).

\section{Conclusiones}

A lgunas especies problemáticas en otras regiones de Ecuador, todavía no tienen una elevada frecuencia de aparición en las zonas periurbanas de Puyo. Las causas más probables son principalmente dos. La primera es la capacidad de adaptación concreta de cada especie a este clima con altas precipitaciones y pocas horas de luz al día. Por ejemplo, el tulipán africano es muy invasor en los ambientes tropicales secos; en el área estudiado existe en al menos tres lugares separados dentro de la ciudad, donde fueron introducidos deliberadamente en zonas ajardinadas y en la rivera de un arroyo del centro de la ciudad y los árboles ya tienen un buen porte, lo que sugiere que esos ejemplares viven desde hace más de 15-20 años, pero en sus alrededores no existen plántulas $\mathrm{o}$ individuos jóvenes.

La observación periódica de estos árboles nos indica que la floración sí se produce, pero estas flores caen con las lluvias frecuentes de la zona y no producen frutos con semillas. La segunda causa parece ser debida a su reciente llegada a la $\mathrm{AAE}$, que es el caso del Ricino o higuerilla, la caña de Castilla o el ojo de venus, las cuales existen tan solo en uno o dos sitios de todo el área periurbana y su porte sugiere una reciente introducción, por ello aún no están extendidas; pero parecen prosperar correctamente en este clima y seguramente sí pasarán a ser problemáticas en la $\mathrm{AAE}$ con el paso del tiempo, por eso se recomienda ser tenidas en cuenta por las autoridades ambientales y prohibir o al menos desaconsejar su uso como planta ornamental.

Entre los vegetales invasores potenciales más preocupantes se encuentra $R$. niveus, cuyas semillas es dispersada por aves y capaz de invadir rápidamente campos de cultivos (Herrera et al., 2016); y la especie M. capitulata, que aunque actualmente tampoco es una planta muy frecuente en la zona estudiada, ya que se encuentra a menudo como ornamental por sus llamativas hojas listadas, por ello, no tardará en naturalizarse e instalarse en la Amazonía Ecuatoriana como ya ha sucedido en Colombia y Brasil.

Aunque en estas latitudes normalmente carezca de semillas viables, su potencial como plaga en un futuro próximo es muy alto, ya que en nuestro clima tropical húmedo se reproduce muy rápido por medio de rizomas y desplaza sin problemas a la vegetación natural, por lo tanto consideramos que debe evitarse su plantación en la toda la AAE y alentamos a que las autoridades ambientales consideren posibles medidas en la divulgación, control e incluso la erradicación total de esta planta y de las otras tres especies reflejadas en la Cuadro 2, ya que se debe tener en cuenta la rapidez de su extensión y los problemas que causan en otras regiones de mundo si no son erradicadas a tiempo.

El Puyo, a pesar de haberse establecido como ciudad hace 120 años aproximadamente, su zona periférica 
presenta 15 especies exóticas invasoras del mundo de acuerdo al listado del GEEI, de las cuales: Molineria capitulata, Hedychium coronarium, Eichhornia crassipes y Rubus niveus presentan un potencial comportamiento a ser invasoras, las cuales han ingresado principalmente por uso ornamental siendo el origen mundial el continente asiático a excepción de $E$. crassipes que es de origen sudamericano.

\section{Bibliografía}

Aguilar, V. 2005. Especies invasoras: una amenaza para la biodiversidad y el hombre. CONABIO. Biodiversitas: 7-10

Altamirano, C., Cueva, E. 2011. Estudio y Experimentación de Paneles Estructurales y de revestimiento en Base de la Caña de la Sierra. Facultad fr Arquitectura de la Universidad de Cuenca.

Buddenhagen, G.E. 2006 The successful eradication of two blackberry species Rubus megalococcus and $R$. adenotrichos (Rosaceae) from Santa Cruz Island, Galapagos, Ecuador. Pacific Conservation Biology 12: 272-278 (20)

CABI. 2015. Eichhornia crassipes. En: Invasive Species Compendium. Wallingford, UK: CAB International. Consultado en marzo 2018. En http://www.cabi.org/isc/ datasheet/108967

CDB. 1992. Convenio sobre la Diversidad Biológica. Río de Janeiro, Brasil. En: https://www.cbd.int/convention/text/ default.shtml

Coetzee, J., Hill, M., Hurtado, A., Laranjeira, C., Nang'alelwa, M., Ruíz, T. T., Schrader, G., Starfinger, U. \& Julien, M. 2008. Report of a Pest Risk Analysis: Eichhornia crassipes. European and Mediterranean Plant Proteccion Organization. Consultado en marzo 2018 en Http:// www.eppo.int/QUARANTINE/Pest_Risk_Analysis/ PRAdocs_plants/08-14408_PRAreport_Eichhornia.pdf

Crespo, C. 2012. Simulación herbívora para un potencial agente defoliador de Rubus niveus en las Islas Galápagos. Trabajo de graduación, previo a la obtención del título de Biólogo del Medio Ambiente. Universidad del Azuay.

Endress, P.K. 1995. Diversity and Evolutionary Biology of Tropical Flowers. Cambridge University Press, Cambridge, England. 311 pp.

Farnese, F.S; J.A. Oliveira; Lima, F.S; G.A. Leão; Gusman, G.S; L.C. Silva. 2013. Evaluation of the potential of Pistia stratiotes L. (water lettuce) for bioindication and phytoremediation of aquatic environments contaminated with arsenic. Braz. J. Biol., 2014, vol. 74, no. 3 (suppl.), p. S103-S112.

Flynn, T. \& Lorence, DH (2002). Las adiciones a la flora de las islas de Hawai. Bishop Museum documentos ocasionales 69:

Francis, John K. 1993. Bambusa vulgaris Schrad ex Wendl.
Common bamboo. SO-ITF-SM 65. New Orleans, LA: U.S. Department of Agriculture, Forest Service, Southern Forest Experimental Station. 6 p.

González-Castillo, J.C., Hahn Von-Hessberg, C.M. \& Narváez-Solarte, W., 2014. Características botánicas de Tithonia diversifolia (Asterales: Asteraceae) y su uso en la alimentación animal. Bol. Cient. Mus. Hist. Nat. U. de Caldas, 18 (2): 45-58.

Grapas, GW, Herbst, DR. 2005. Un Jardín Tropical flora - Las plantas cultivadas en las islas de Hawai y otros lugares tropicales. Bishop Museum Press, Honolulu, HI.

Hernández J. 2008. Especies exóticas invasoras como agentes de cambio global. En: Schüttler, E. \& Karez, C.S. (eds). Especies exóticas invasoras en las Reservas de Biosfera de América Latina y el Caribe. Un informe técnico para fomentar el intercambio de experiencias entre las Reservas de Biosfera y promover el manejo efectivo de las invasiones biológicas. UNESCO, Montevideo

Herrera, I., 2016. Distribución potencial y asignación de riesgo para las plantas terrestres exóticas identificadas oficialmente en el Ecuador. Catalogo descriptivo de especies exóticas. http://repositorio.educacionsuperior. gob.ec/handle/28000/4950

Herrera, I., Goncalves, E., Pauchard, A. Bustamante, R.O. 2016. Manual de plantas invasoras de Sudamérica. Santiago de Chile. ISBN 978-956-362-998-9

Landázuri, O. 2010. La 'mora' Rubus niveus, algunos datos importantes sobre la especie en el contexto de la problemática de control y erradicación de la especie. La Granja. Vol.12 (2). Pp. 28-31. ISSN: 13903799

Leonar. E.C. 1958. The Acanthaceae of Colombia. Contr. U.S. Natl. Herb. 31:1-781.

López, D. 2012. “Aprovechamiento de lechuguin (Eichhornia crassipes) para la generación de abono orgánico, mediante la utilización de tres diseños diferentes de biodigestores. Tesis de grado. Universidad Politécnica Salesiana. Carrera de Ingeniería Ambiental.

Lowe, S., Browne M., Boudjelas S., De Poorter M. 2000. 100 of the World's Worst Invasive Alien Species. A selection from the Global Invasive Species. Published by The Invasive Species Specialist Group (ISSG) a specialist group of the Species Survival Commission (SSC) of the World Conservation Union (IUCN), 12pp.

Matienzo, Y., Ramos, B., Rijo, E. 2003. Revisión bibliográfica sobre lantana camara 1. una amenaza para la ganadería. fitosanidad [en linea], 7 (Diciembre-Sin mes) : [Fecha de consulta: 11 de septiembre de 2017] Disponible en: <http://www.redalyc.org/articulo. oa? id=209118173010> ISSN 1562-3009.

Mendoza, R. y P. Koleff (coords.). 2014. Especies acuáticas invasoras en México. Comisión Nacional para el Conocimiento y Uso de la Biodiversidad, México

Ministerio del Ambiente MAE, 2011. Lista preliminar de especies exóticas introducidas e invasoras en el Ecuador 
continental. Quito

Ojasti, J. 2001. Estudios sobre el Estado Actual de las Especies Exóticas. Comunidad Andina/ Banco Interamericano de Desarrollo. Caracas, Venezuela. 223 pp.

Pereda, J.; Cambero, M.; García, M. 1998. Tecnología de los alimentos: Componentes de los alimentos y procesos. Vol. I, Edit. Síntesis (España). 365 p.

Rico, R., Tapia, L., Teniente, R., González A., Hernández, M., Solís, L., Zamarripa, A. 2011. Guía para cultivar higuerilla (Ricinus communis L.) en Michoacán. Instituto Nacional de Investigaciones Forestales, Agrícolas y Pecuarias, Centro de Investigación Regional del Pacífico Centro, Campo Experimental Valle De Apatzingán. Apatzingán, Michoacán, México. Folleto Técnico Núm. 1.

Ríos, A; J. Aristegui; Frondoy, L y M. Gómez. 2012. Consideraciones para el conocimiento de yerba carnicera (Conyza spp). La Estanzuela, INIA

Santos, S.B., Pedralli, G., Meyer, S.T. 2005. Aspectos da fenologia e ecologia de Hedychium coronarium (Zingiberaceae) na estação ecológica do Tripuí, Ouro Preto-MG. Planta Daninha, 23(2), 175-180. https:// dx.doi.org/10.1590/S0100-83582005000200002

Segovia C, Quijia P, Proaño K, Soltis P y Soltis D. 2010. La Desaparición de los Bosques de Polylepis (Rosaceae: Rosoideae: Sanguisorbeae) en el Ecuador: Hibridización y las implicaciones para su manejo y conservación. Ecociencia. Quito.

Soria, M., Taylor, U., Tye, A., Wilkinson, S.R. 2002. Manual de identificación y manejo de malezas en Galápagos. Charles Darwin Research Station, Puerto Ayora, Galapagos, Ecuador. 66 pp.”

Stone, C. P.; Smith, C.W.; Tunison, J.T. 1992. Alien plant invasions in native ecosystems of Hawaii: management and research. Honolulu: University of Hawaii, Cooperative National Park Resources Study Unit, 1992. p. 887.

The Catalogue of Life Partnership. 2017. Catalogue of Life. Checklist Dataset https://doi.org/10.15468/rffz4x accessed via GBIF.org on 2017-09-11.

Trombulak, S.C., Frissell, C.A. 2000. Review of ecological effects of roads on terrestrial and aquatic communities. Conservation Biology 14:18-30.

Tunison, T. 1991. Element stewardship abstract for Rubus argutus, the Nature Conservancy, Virginia, USA. Revisions

Valaezo, J. 2013. Rendimiento y valoración nutritiva de especies forrajeras arbustivas establecidas en bancos de proteína, en el sur de la Amazonía ecuatoriana. Universidad Nacional de Loja. Centro de Estudios y Desarrollo de la Amazonía (CEDAMAZ) Dirección de investigaciones. Revista CEDAMAZ. Volumen 3, No. 1

Verdejo, E., Palmerín, J. A., Aibar, J., Cirujeda, A., Taberner, A., Zaragoza, C. 2006. El lirio de Agua Eichhornia crassipes. Plantas Invasoras. Ministerio de Agricultura, Pesca y Alimentación. Secretaría General Técnica. 7pp

Williamson, M.H., Fitter, A. 1996. The characters of successful invaders. Biological Conservation; 78(12):163-170.

Zambrano, L.F., Buenaño, M.P., Mancera, N.J., Jiménez, E. 2015. Estudio etnobotánico de plantas medicinales utilizadas por los habitantes del área rural de la Parroquia San Carlos, Quevedo, Ecuador. Rev Univ. salud; 17(1): $97-111$ 